\title{
Egg removal and intraspecific brood parasitism in the European starling (Sturnus vulgaris)
}

\author{
M.P. Lombardo, H.W. Power, P.C. Stouffer, Linda C. Romagnano, and Ann S. Hoffenberg \\ Department of Biological Sciences, Rutgers University, P.O. Box 1059, Piscataway, NJ 08855-1059, USA
}

Received April 18, 1988 / Accepted January 6, 1989

Summary. From 1983 to 1986 we monitored 284 European starling (Sturnus vulgaris) nests in New Jersey for evidence of intraspecific brood parasitism and egg removal during the laying period. Egg removal occurred significantly more often at nests where intraspecific brood parasitism was detected (12 of 35 nests, $34 \%$ ) than at unparasitized nests (23 of 249 nests, 9\%). Brood parasitism $(92 \%$ of parasitized nests) and egg removal (74\% of nests with egg removal) were most common at nests where egg laying began in April of each year (i.e., early nests). Egg removal occurred at $26(19 \%)$ and brood parasitism at $32(23 \%)$ of 138 early nests. Both brood parasitism and egg removal were concentrated during the first four days in the laying period when brood parasitism is most likely to be successful and when host nests are most vulnerable to parasitism (Romagnano 1987). Both parasitism and removal usually involved a single egg at each nest. We detected brood parasitism and egg removal on the same day at five of 12 nests $(42 \%)$ where both were observed. Because starlings do not remove foreign eggs from their nests once they begin laying (Stouffer et al. 1987) we hypothesize that parasite females sometimes removed host eggs while parasitizing nests.

\section{Introduction}

Egg loss and destruction are often associated with intraspecific brood parasitism (e.g., Weller 1959; Victoria 1972; Yom-Tov et al. 1974; Vehrencamp

Present address and address for offprint requests: M.P. Lombardo, Museum of Zoology. Department of Biology, and Michigan Society of Fellows, University of Michigan, Ann Arbor, MI 48109-1079, USA
1978; Evans 1980, 1988; Karlsson 1983, in Andersson 1985; Brown 1984, 1988; Feare 1984; Emlen and Wrege 1986; Frederick 1986; Møller 1987; Brown and Brown 1988). Frequently such egg loss is the result of the physical struggle between host and parasite over priority at the nest (e.g., see Weller 1959; Brown and Brown 1988). In the European starling (Sturnus vulgaris) (Karlsson 1983, in Anderson 1985; Stouffer et al. 1987), whitefronted bee-eater (Merops bullockoides) (Emlen and Wrege 1986), and barn swallow (Hirundo rustica) (Møller 1987) breeding females guard against parasitism by removing foreign eggs from their nests before they, themselves, begin laying.

We examined the association between egg removal and intraspecific brood parasitism in the European starling because egg removal is an hypothesized correlate of brood parasitism in this species (Yom-Tov et al. 1974; Yom-Tov 1980; Evans 1980, 1988; Karlsson 1983, in Andersson 1985; Feare 1984). Various observers have suggested that parasite female European starlings (Evans 1980, 1988; Karlsson 1983, in Andersson 1985; Feare 1984) behave like some interspecific brood parasites and remove host eggs when parasitizing nests (e.g., cowbirds, Friedman 1929; Old World cuckoos, Payne 1977 a).

\section{Methods}

From 1983 to 1986 we studied starlings that nested in boxes mounted on utility poles along the roadways of the Kilmer Campus of Rutgers University in Piscataway, Middlesex Co., New Jersey. Thirty next boxes were available in 1983, 50 in 1984, 75 in 1985, and 68 in 1986. The site has supported a breeding population of starlings in next boxes since 1975 (Crossner 1977). The study site contained mowed lawns, old fields, and parking lots. To prevent racoons (Procyon lotor) and gray squirrels (Sciurus carolinensis) from destroying nests, each pole was wrapped with $0.70 \mathrm{~m}$ wide aluminum flashing below the box. 
In 1983 and 1984, we visited nests once in the morning and again in mid-afternoon during the laying period. In 1985 and 1986, we visited nests three times a day during the laying period. Usually the first census of the day began and was completed between 0700 and 0800 EST, the second between 1100 and 1200 EST, and the third between 1500 and 1700 EST. Our first census was conducted before 0800 because only $17.6 \%$ of female starlings have laid eggs at this time (Feare et al. 1982; Romagnano 1987). Thus, if two or more new eggs were discovered during the second or third census, only one could be a host egg (e.g., Woodward and Mather 1964; Fraps 1965; Gilbert 1971; Feare 1984). Eggs were numbered in sequence with indelible ink. In all years, each nest was monitored until clutch completion. To avoid needlessly disturbing the birds, nests were not usually visited again until one day before its eggs were expected to hatch.

We used a combination of frequent nest censuses and polyacrylamide gel electrophoresis to detect intraspecific brood parasitism (Romagnano 1987). Censusing occurred in all years but the use of electrophoresis was restricted to 1983-1985. Breeding females and males were usually captured six to seven days after the eggs hatched, uniquely banded with U.S. Fish \& Wildlife Service numbered aluminum bands and color bands if not already banded, and biopsied for pectoral muscle and blood for electrophoretic analysis. Chicks were banded and biopsied 20 days after they hatched. Hoffenberg et al. (1988) and Romagnano (1987) describe these techniques in detail.

A nest was defined as parasitized if (1) more than one egg appeared within $20 \mathrm{~h}$ (no bird species is known to lay more than one egg per $24 \mathrm{~h}$ period; Woodward and Mather 1964; Fraps 1965; Gilbert 1971; Feare 1984), or (2) the electromorphs of blood plasma and pectoral muscle of one or more nestlings did not match the electromorphs of their putative mother's (Romagnano 1987).

An egg was considered removed if it disappeared from a nest after being marked. We briefly searched the ground beneath and between nest boxes for eggs during all censuses. Although eggs also disappeared from nests during the post-laying incubation period they are not included in the analyses that follow because we did not census nests frequently enough during incubation to be able to confidently attribute the removal of an egg from a nest to resident or non-resident starlings.

\section{Results}

Egg removal occurred at $12 \%$ of the 284 nests monitored during the laying period (Table 1). Removal occurred significantly more often at nests where parasitism was detected (12 of $35,34 \%)$ than at nests where it was not $(23$ of $249,9 \%)\left(\chi^{2}=\right.$ 16.12, $d f=1, P<0.001)$. However, the analyses that follow are restricted to the 138 early broods because (1) nests where egg laying was initiated in April of each season represented a highly visible peak of reproductive synchrony (Romagnano 1987), (2) $92 \%$ of the nests with brood parasitism and $74 \%$ of the nests with egg removal were early broods, and (3) the high mortality of late clutches due to causes unrelated to brood parasitism (e.g. ectoparasite infestation of nestlings) made it difficult to biopsy birds before nest failure and thus evaluate the relationship between brood parasitism
Table 1. Intraspecific brood parasitism and egg removal at starling nests in New Jersey. Numbers in parentheses are the number of parasite or removed eggs

\begin{tabular}{|c|c|c|c|c|}
\hline $\begin{array}{l}\text { Month } \\
\text { egg-laying } \\
\text { initiated }\end{array}$ & Nests & $\begin{array}{l}\text { Para- } \\
\text { sitized } \\
\text { nests }\end{array}$ & $\begin{array}{l}\text { Nests } \\
\text { with egg } \\
\text { removal }\end{array}$ & $\begin{array}{l}\text { Nests with } \\
\text { parasitism and } \\
\text { egg removal }\end{array}$ \\
\hline April & 138 & $32(43)$ & $26(29)$ & 11 \\
\hline May & 91 & 1 (1) & $4(6)$ & 0 \\
\hline June & 55 & $2(2)$ & $5(5)$ & 1 \\
\hline Total & 284 & $35(46)$ & $35(40)$ & 12 \\
\hline
\end{tabular}

and egg removal. Nestlings survived to biopsy age at only two of the nine nests where we observed egg removal during laying in May and June (Table 1).

Feare (1984) suggested that the disturbance resulting from investigator visits to starling nests might cause an increase in the frequency of egg removal. However, in this study egg removal at early nests was independent of whether we made two $(9$ of $62(15 \%)$ nests had removal) or three (17 of $76(22 \%)$ nests had removal) censuses a day $\left(\chi^{2}=1.37, d f=1, P>0.10\right)$.

Twenty-nine eggs were removed from 26 to 138 $(19 \%)$ early broods (Table 1$)$. This result corresponds well to the proportion of nests (12 of 52 nests, $23 \%$ ) at our study site where Kennedy et al. (1989) using macroscopic examination discovered that the number of postovulatory follicles (POFs) (i.e., the split, yellowish chambers that remain in a bird's ovary after ovulation) in a resident female's ovary exceeded the number of eggs in her nest. Because a macroscopic examination of POFs provides a direct index of true clutch size (Kennedy et al. 1989, references therein), Kennedy et al. (1989) inferred that most cases in which POFs exceeded the number of eggs were likely due to egg removal.

We found six numbered eggs on the ground directly below boxes during census visits. We have observed starlings dropping eggs from the nest box hole (this study; Stouffer et al. 1987). Two of these eggs had holes in their sides that approximated the size of a starling's bill. Two eggs had large holes in their sides and it appeared as though the albumin in each egg had been eaten. The remaining two numbered eggs were undamaged. Twentythree eggs disappeared from clutches between census visits and were not found.

We found 30 unnumbered eggs on the ground near to or directly below nests during potential host laying periods. Nine unnumbered eggs were found below six different nests before the resident 
females at those nests began laying. We hypothesize that these eggs were laid by parasite females but were removed by resident females. Resident female starlings quickly removed foreign eggs placed in their nests by Stouffer et al. (1987).

Sixteen unnumbered eggs were found either within $15 \mathrm{~m}$ of $(n=3)$ or directly below $(n=13)$ 14 different nests during the laying periods of the resident females at those nests. The distribution of the ratios of unnumbered to numbered eggs found on the ground each day during laying (Table 2) was not significantly different (Log Likelihood Ratio, $\mathrm{G}=0.846, d f=6, P>0.25$ ) from that expected given the following assumptions: (1) Parasites and hosts, on average, laid eggs between our 0700 and 1100 census each day (Romagnano 1987) meaning that one unnumbered egg was in a nest when the parasite came and removed an egg. (2) Each egg in a nest had the same probability of being removed. We hypothesize that these unnumbered eggs were hosts' eggs that were removed by parasites before we had a chance to number them (i.e., they were removed between censuses). Twelve of these $16(75 \%)$ unnumbered eggs appeared at nests between our 0700 and 1100 censuses. It is unlikely that host females removed these eggs because resident starling females do not remove foreign eggs placed in their nests once they begin laying (Stouffer et al. 1987).

At four of these $14(29 \%)$ nests we found a newly laid egg in the nest during the same census when an unnumbered egg was found on the ground. At three of these $14(21 \%)$ nests we found a newly laid egg in the nest during the census immediately after an unnumbered egg was found on the ground. Given that resident female starlings do not remove foreign eggs placed in their nests after they begin laying (Stouffer et al. 1987), this observation that newly laid eggs were found either simultaneously with or after the discovery of unnumbered eggs at 50 percent of these 14 nests suggests that parasite females sometimes remove host eggs while parasitizing nests. However, we were unable to determine whether these eggs were parasite eggs using electrophoresis (Romagnano 1987). This was not surprising given the conservatism of electrophoresis in our population (Romagnano 1987).

We found five unnumbered eggs on the ground near four nests during circumstances difficult to evaluate. One unnumbered egg was found equidistant between two nests making it impossible to assign it a nest of origin. One unnumbered egg was found under a box in which two females were nesting communally (see Stouffer et al. 1988). The
Table 2. The distributions of egg removal, unnumbered eggs found on the ground, and brood parasitism during host laying periods at 138 early starling nests. Laying Day $1(\mathrm{LD} 1)=$ the day the first egg is laid

\begin{tabular}{lrrrrrrrrr}
\hline & \multicolumn{1}{c}{ Day in the laying period } \\
\cline { 2 - 9 } & 1 & 2 & 3 & 4 & 5 & 6 & 7 & 8 & Total \\
\hline Eggs removed & 5 & 6 & 5 & 6 & 5 & 1 & 1 & 0 & 29 \\
Unnumbered eggs found 5 & 4 & 2 & 0 & 4 & 1 & 0 & 0 & 16 \\
Parasite eggs laid & 10 & 13 & 10 & 4 & 4 & 1 & 1 & 0 & 43 \\
\hline
\end{tabular}

ejection of eggs from communal nest by females laying at that nest is common (e.g., see Vehrencamp 1977; Bertram 1979; Mumme et al. 1983). One unnumbered egg was found under a nest two days after clutch completion at that nest. Two unnumbered eggs were found under a single nest. Each was found on successive days, four and five days after a single egg was laid in that nests. We observed a male remove one of these eggs.

Because unnumbered eggs were unmarked, we cannot exclude the possibility that they were laid on the ground by parasites that were unable to find unguarded nests (cf. Evans 1988). However, we do not think that this is likely because most of the unnumbered eggs ( 26 of 30 eggs, $87 \%$ ) were found directly below nest box holes suggesting that they were dropped from the hole.

We detected brood parasitism at $23 \%$ of early nests (Table 1) using both census and electrophoretic techniques (Romagnano 1987). For parasitism to be a successful strategy a parasite egg must have a high probability of producing a fledgling (Hamilton and Orians 1965). For our population this means that parasite eggs must be laid before the initiation of incubation (Romagnano 1987). Parasite eggs laid after this time hatch late and produce runts with low chances of fledging relative to eggs that hatch on schedule (Litovich 1982). The modal date of the initiation of incubation in our population is Laying Day 4 (LD1 = the day the first egg was laid. Thirty-seven of the $43(86 \%)$ parasite eggs that we detected were laid before Laying Day 5 (Table 2). This proportion was not significantly different from the proportion of the eggs removed before LD5 (22 of 29 eggs; $76 \%$ ) $\left(\chi^{2}=1.21, d f=1, P>0.25\right)$ or the proportion of unnumbered eggs that we found on the ground before LD5 (11 of 16 eggs; $69 \%) \chi^{2}=1.31, d f=1, P>$ $0.25)$.

At 24 of the $26(92 \%)$ nests where eggs were removed only a single egg was removed. Likewise, multiple parasitism was also rare (Romagnano 1987). Twenty-three of the $32(72 \%)$ nests where 
Table 3. The frequencies of multiple acts of removal and multiple parasitism at early nests. Single acts of removal were more common than single acts of parasitism $\left(\chi^{2}=4.54, d f=1, P<\right.$ 0.05 ). The number in parentheses are the number of nests observed in each category if nests III-10 and III-17 from 1985 are eliminated from the analysis (see text). Analyzed this way single acts of removal and parasitism are equally common $\left(\chi^{2}=\right.$ $2.53, d f=1, P>0.10)$

\begin{tabular}{llll}
\hline & One egg & $\begin{array}{l}\text { More than } \\
\text { one egg }\end{array}$ & Total \\
\hline Parasitism & 23 & $9(7)$ & $32(30)$ \\
Removal & $24(23)$ & 2 & $26(25)$ \\
\hline
\end{tabular}

we detected parasitism had a single parasite egg. The removal of a single egg was more common than a single act of parasitism $\left(\chi^{2}=4.54, d f=1\right.$, $P<0.05$ ) (Table 3 ). However, if we eliminate two nests monitored in 1985 (III-10 and III-17) from this analysis, the removal of a single egg was as common as a single act of parasitism $\left(\chi^{2}=2.53\right.$, $d f=1, P>0.10)$ (Table 3 ). We justify removing these nests from the analysis because it appeared as though two separate parasites "staked out" each of these nests. Therefore, nests III-10 and III17 were not typical parasitized nests. At III-10, one parasite egg was laid each morning on LD1LD3. These parasite eggs were readily distinguishable from the host eggs by color. No eggs were removed from III-10. At III-17, one parasite egg was laid each morning on LD2-LD4. Egg one was removed before 0758 on LD2 (Table 4). The parasite eggs were lighter than the host eggs. Both the color-detected series at III-10 and the weight-detected series at III-17 were internally homogeneous implying a single brood parasite at each nest.

At five of the 12 nests (42\%) where both egg removal and brood parasitism were detected, both events occurred on the same day (Table 4). By using clutch size as an indicator of the number of days available for both egg removal and parasitism, and assuming that egg removal and parasitism were independent events, we determined the probability of both of them occurring on the same day at any one of the 12 nests where both were detected by using the binomial expansion,

$$
\frac{\mathrm{N} !}{\mathrm{X} ! \mathrm{Y} !}(\mathrm{X})^{\mathrm{p}}(\mathrm{Y})^{\mathrm{q}}
$$

where $\mathrm{N}$ is the number of laying days for a clutch (e.g. if clutch size was five there were five laying days, $n=5$ ), $\mathrm{X}=$ the number of days on which both removal and parasitism could occur on the same day, $\mathrm{Y}=$ the number of days during which removal and parasitism could occur on different days $(\mathrm{N}-$
Table 4. Nests where egg removal and intraspecific brood parasitism were both detected. The Nest category lists the year, then the nest box. The day the first egg was laid is equal to day one (LD1). Egg number is symbolized by En, where n is the egg's position in the laying sequence

\begin{tabular}{ll}
\hline Nest & $\begin{array}{l}\text { Removal } \\
\text { in relation } \\
\text { to parasitism }\end{array}$ \\
\hline
\end{tabular}

April nests
84-III-5 2 days after

E2 and E3 appeared on LD2. E4 was removed by 0800 on $\mathrm{LD} 4$ and $\mathrm{E} 5$ was laid by 1130 on the same day

84-I-10old Same day

E5 a parasite egg detected by electrophoresis was laid between 0730 and 1157 on LD5. E2 was removed between 1157 and 1713 on LD5

85-I-12old Same day E2 a parasite egg detected by electrophoresis was laid between 0749 and 1147 on LD2. E1 was removed during the same time interval

85-I-18old 1 day before E1 was removed on LD3. E2, 3, 4 and were laid on LD4. E4 was found 2 days after dented on LD5 and removed on LD6

85-I-20old 2 days after E2 a parasite egg detected by electrophoresis was laid on LD2 E3 was removed between 1124 and 1648 on LD4

85-III-9 1 day after E1 was a parasite egg detected by electrophoresis. E2 was laid between 0735 and 1133 on LD2. E2 was removed between 1133 and 1604 on LD2

85-III-17 1 day before E1 was removed by 0758 on LD2. E2 and E3 were laid between 0758 and 1214 on LD2.

Two other parasite eggs were laid on LD3 and LD4

86-I-20 3 days after E1 and E2 were laid on LD1. E5 was and laid between 0828 and 1152 and 1 day before removed between 1152 and 1523 on LD4. Two new eggs were laid on LD5

86-II-6 1 day before E2 was removed by 0835 on LD3 and was replaced by a new unmarked egg. Two new eggs were laid on LD4

86-II-7 Same day On LD1 two starlings were observed entering and leaving the nest before our inspection at 0938 revealed two eggs, one of which was punctured. The punctured egg was removed before 1241 on LD1

86-III-2 Same day On LD5, E5 was laid by 1132. By the next census at $1611, \mathrm{E} 1$ was removed and E6 was laid

June nests

85-II-7 Same day On LD2, E1 was missing and two new unmarked eggs were found in the nest 
$\mathrm{X}=\mathrm{Y}), \mathrm{p}=$ the probability of parasitism and removal occurring on the same day at each nest of given clutch size $\mathrm{N}$ (e.g., if $N=5$, the probability of removal occurring on any one day $=1 / 5$ and the probability of removal occurring on any one day $=$ $1 / 5$, thus the probability of both occurring on any day ( $a$ through $e$ ) within the laying period at that nest $=1 / 25$ ), and $q=$ the probability of removal and parasitism occurring on different days in the laying period, 1-p. Throughout we assume that both removal and parasitism result in either one egg being removed from or added to a clutch (Table 3 ).

We compared the expected number of nests where both removal and parasitism should have been detected on the same day to the observed number using the Chi-square Goodness of Fit Test (Zar 1974). This comparison $\left(\chi^{2}=10.87, d f=4\right.$, $P<0.05)$ suggests that removal and parasitism were related phenomena. However, because of the small sample size it is not safe to make this inference in the absence of additional information.

The additional information necessary to link egg removal and brood parasitism was found by examining the chronology of removal and parasitism at the nests where both were detected (Table 4). Interspecific brood parasites sometimes remove eggs one day before they parasitize host nests (Friedman 1929; Payne 1977 a). A very important piece of information in determining the identities of the birds (hosts or parasites) that removed eggs, is the fact that host starlings in our population do not remove foreign eggs added to their nests once they have begun laying (Stouffer et al. 1987). Karlsson (1983, in Andersson 1985) obtained the same results in a similar experiment on starlings in Sweden. Therefore, eggs removed from nests were probably removed by the parasites and not by the hosts.

\section{Discussion}

Egg removal was common during the laying period at starling nests in New Jersey. There was a chronological and statistical association between removal and parasitism. Evans' (1980, 1988) observed that egg removal was more frequent at parasitized nests than at nests where parasitism was not detected at his study sites in Great Britain. The sequence of events at nests where removal and parasitism were both detected suggested that parasites sometimes removed host eggs at the nests they parasitized. However, there are several alternative explanations for the phenomenon of egg removal.

First, egg removal can be the result of egg predation by mammals or other bird species. How- ever, it is very unlikely that predators were responsible for the egg removal that we observed because (1) since installing predator guards in 1979 no nests have failed because of predation, (2) mammalian predators usually take a whole clutch and leave the nest in disarray by pulling the nest material out of the nest box hole (Litovich 1982); there was no evidence of mammalian predation at nests with egg removal, and (3) it is unlikely that egg removal was caused by other bird species (e.g., house wrens, Troglodytes aedon, or house sparrows, Passer domesticus) known to destroy eggs because no other species nested in our boxes or were seen entering them.

Second, egg removal can be the result of usurpation tactics by other starlings. Starlings are nonexcavating cavity nesters and must rely on preexisting cavities to breed. Therefore, they are likely limited by nest sites. The inter- and intraspecific nest site usurpation strategies of other non-excavating cavity species (e.g., tree swallow, Tachycineta bicolor; house wren, eastern bluebird, Sialia sialis; house sparrow) are well known (see Kuerzi 1941; Bent 1942, 1948, 1949; Summers-Smith 1963; Leffelaar and Robertson 1985; Belles-Isles and Picman 1986). A common tactic in their usurpation strategies is the removal or destruction of resident eggs. A nest usurper can best precipitate a resident's nest failure and abandonment by removing all of the resident's eggs or by removing as many eggs as possible. However, single eggs were most often removed from nests during laying and egg removal during laying was not associated with nest abandonment in this study.

Egg removal ( $n=84$ eggs) also occurred during the post-laying incubation period at 35 of 284 $(12 \%)$ total nests, and 14 of $138(10 \%)$ early nests. At five of these $14(36 \%)$ early nests, complete clutches were removed, consistent with a hypothesis that nest usurpers were responsible for most of the removal that occurred during the post-laying incubation period. However, we did not visit nests frequently enough once clutches were complete to exclude alternative hypotheses to explain removal during the post-laying incubation period.

Third, egg removal can be the result of hosts removing parasite eggs that they discover in their nests. Evans (1988) hypothesized that the reduced breeding success at parasitized nests at his study sites in Great Britain resulted from females removing eggs from their nests when their clutches were small. However, the results of experiments performed by Stouffer et al. (1987) on our population of starlings do not support Evans' hypothesis. Stouffer et al. (1987) found that hosts did not dis- 
criminate between their own and parasite eggs once they, themselves, began laying. A similar lack of discrimination after egg laying has been reported in other species (Rothstein 1975; Lanier 1982; Brown 1984; Emlen and Wrege 1986; Møller 1987). Thus, it is extremely unlikely that host females removed parasite eggs added to their partial clutches. In addition, the electrophoretic data (Romagnano 1987) show that some of the eggs that were not removed were parasite eggs. Therefore, we conclude that resident females did not remove eggs from their own nests during the laying period during this study and that parasite females sometimes removed host eggs at the nest that they parasitize. That egg removal sometimes occurred after parasitism (Table 4) is difficult to explain because we have no a priori reason to expect that parasite females can discriminate their own eggs from host eggs (cf. Stouffer et al. 1987). The sequence of events at the five nests where removal occurred after parasitism make it difficult to associate removal with parasitism in a coherent manner without making undue assumptions.

We may be underestimating the number of nest usurpation attempts and overestimating the number of potential parasites because some of the eggs removed during egg laying may have been removed by would-be nest usurpers. For example, if a prospective nest usurper removed one or more eggs from a nest soon after the resident began laying, but the resident completed her clutch, we would assign this pattern of removal to parasites. This scenario suggests another alternative reproductive strategy. The nonresident female in this scenario may remove eggs from a nest and if the resident does not desert she parasitizes it, but if the resident abandons her nest, the non-resident usurps the nest.

The patterns of egg removal and brood parasitism suggest the tactics that may be used by brood parasites. Both egg removal and the appearance of parasite eggs were concentrated early in the laying period. This short window of vulnerability/opportunity (LD1-LD4) also suggests why the number of cases of multiple parasitism were rare (Romagnano 1987). A parasite must lay her egg in a host nest after the host has begun laying (Stouffer et al. 1987) but before LD5. Therefore, the periods of parasite opportunity and nest vulnerability are short (Romagnano 1987). Likewise, Evans (1988) found that parasite eggs were more likley to produce fledglings when they were laid synchronously with host eggs. Apparently, parasitic cliff swallows (Hirundo pyrrhonota) are not faced with the same time constraints as starling parasites.
Brown and Brown (1988) report that the eggs removed from cliff swallow nests as a prelude to having eggs transferred into them (Brown 1988) were removed during incubation and after the end of laying. Interspecific brood parasites often are not faced with the time constraints challenging starling parasites (Payne 1977a).

The laying of a single parasite egg in each of several host nests is a tactic commonly employed by intraspecific brood parasites (e.g., see Evans 1980, 1988; Brown 1984, 1988; Emlen and Wrege 1986; Møller 1987). By spreading her eggs over several nests, the parasite (1) minimizes the time spent at each nest thereby reducing her chances of being detected, (2) keeps the host clutches from exceeding the sizes that the host can rear and thereby increases the chances that her chick will fledge, and (3) increases the chances that some of her young will fledge by not having all of her eggs in one potentially doomed nest (Payne 1977a, 1977b; Andersson 1985; Brown 1984, 1988).

The advantages accruing to parasites by spreading their eggs over several nests are augmented by egg removal (Andersson 1985). The egg remover's reproductive success is potentially enhanced at the direct expense of the host. If a nest is parasitized by more than one parasite, a parasite that spreads her eggs over several nests is more likely to remove the eggs of a parasite that targets a specific nest than vice versa (Andersson 1985).

The patterns of egg removal and parasitism that we observed were consistent with Andersson's (1985) hypothesis that parasites should remove single eggs from host nests while laying single parasite eggs in them.

We do not known the identity of the parasite females in our population because unlike Brown $(1984,1988)$ and Emlen and Wrege (1986) we have never observed them laying eggs. Yearling females without nests of their own may represent the largest fraction of the parasite population at our study site (Romagnano 1987), but there may be several classes of female involved (see Yom-Tov 1980; Evans 1980, 1988).

Acknowledgements. We thank K. Cybulski, H. Heinz, T. Vail, and the students in Animal Social Behavior Laboratory 444 at Rutgers University for their help in the field work. C.R. Brown, C.J. Feare, E.D. Kennedy, C.T. Snowdon, R.L. Mumme, R.B. Payne, W.M. Shields, K.E. Linsenmair and an anonymous reviewer provided useful comments on previous versions of the manuscript. T. Tenhave of the Statistical Research Lab at the U. of Michigan helped in determining the statistical association between egg removal and brood parasitism. Our research was supported by NSF grant BSR 8316361 to H.W. Power and T.R. McGuire, and two Busch Memorial Grants from Rutgers University to H.W. Power. Field work 
in 1986 was supported by a Carl N. Steinez Memorial Fund grant from the Department of Biological Sciences of Rutgers University to P.C. Stouffer.

\section{References}

Andersson M (1985) Brood parasites within species. In: Barnard CJ (ed) Producers and scroungers. Chapman \& Hall, New York, pp 195-228

Belles-Isles JC, Picman J (1986) House wren nest-destroying behavior. Condor 88:190-193

Bent AC (1942) Life histories of North American flycatchers, larks, swallows, and their allies. US Nat Mus Bull No 179

Bent AC (1948) Life histories of North American nuthatches, wrens, thrashers, and their allies. US Nat Mus Bull No 195

Bent AC (1949) Life histories of North American thrushes, kinglets, and their allies. US Nat Mus Bull No 196

Bertram BCR (1979) Ostriches recognise their own eggs and discard others. Nature $279: 233-234$

Brown CR (1984) Laying eggs in a neighbor's nest: benefit and cost of colonial nesting in swallows. Science 224:518-519

Brown CR (1988) A new form of reproductive parasitism in cliff swallows. Nature $331: 66-68$

Brown CR, Brown MB (1988) The costs and benefits of egg destruction by conspecifics in colonial cliff swallows. Auk $105 \cdot 737-748$

Crossner KA (1977) Natural selection and clutch size in the European starling. Ecology 58:885-892

Emlen ST, Wrege PH (1986) Forced copulation and intra-specific parasitism: two costs of social living in the whitefronted bee-eater. Ethology 71:2-29

Evans PGH (1980) Population genetics of the European starling (Sturnus vulgaris). PhD thesis, University of Oxford

Evans PGH (1988) Intraspecific nest parasitism in the European starling Sturnus vulgaris. Anim Behav 36:1282-1294

Feare CJ (1984) The starling. Oxford University Press, New York

Feare CJ, Spencer PL, Constantine DAT (1982) Time of egglaying of starlings Sturnus vulgaris. Ibis 124:174-178

Fraps RM (1965) Twenty-four hour periodicity in the mechanism of pituitary gonadotrophin release for follicular maturation and ovulation in the chicken. Endocrinology 77:5-18

Frederick P (1986) Conspecific nest takeovers and egg destruction by white ibises. Wilson Bull $98: 156-157$

Friedman H (1929) The cowbirds. Thomas, Springfield

Gilbert AB (1971) Transport of the egg through the oviduct and oviposition. In: Bell DJ, Freeman BM (eds) Physiology and biochemistry of the domestic fowl, vol 3. Academic Press, New York, pp 1345-1350

Hamilton WJ III, Orians GH (1965) Evolution of brood parasitism in altricical birds. Condor 67:361-382

Hoffenberg AS, Power HW, Romagnano LC, Lombardo MP, McGuire TR (1988) The frequency of cuckoldry in the European starling (Sturnus vulgaris). Wilson Bull 100:60-69

Karlsson J (1983) Breeding of the starling (Sturnus vulgaris). $\mathrm{PhD}$ thesis, University of Lund, Sweden

Kennedy ED, Stouffer PC, Power HW (1989) Postovulatory follicles as a measure of clutch size and brood parasitism in European starlings (Sturnus vulgaris). Condor (in press)
Kuerzi RG (1941) Life history studies of the tree swallow. Proc Linn Soc NY 52-53:1-52

Lanier GA Jr (1982) A test for conspecific egg discrimination in three species of colonial passerine birds. Auk 99:519-525

Leffelaar D, Robertson RJ (1985) Nest usurpation and female competition for breeding opportunities by tree swallows. Wilson Bull 97:221-224

Litovich E (1982) Experiments on the basis of parent-offspring conflict in starlings. PhD thesis, Rutgers University, New Brunswick

Møller AP (1987) Intraspecific nest parasitism and anti-parasite behaviour in swallows, Hirundo rustica. Anim Behav $35: 247-254$

Mumme RL, Koenig WD, Pitelka FA (1983) Reproductive competition in the communal acorn woodpecker: sisters destroy each other's eggs. Nature 306:583-584

Payne RB (1977a) The ecology of brood parasitism in birds. Ann Rev Ecol Syst 8:1-28

Payne RB (1977b) Clutch size, egg size, and the consequences of single vs. multiple parasitism in parasitic finches. Ecology $58: 500-513$

Power HW, Litovich E, Lombardo MP (1981) Male starlings delay incubation to avoid being cuckolded. Auk 98:386-389

Romagnano LC (1987) Intraspecific brood parasitism in the European starling (Sturnus vulgaris). PhD thesis, Rutgers University, New Brunswick

Rothstein SI (1975) Evolutionary parasitism. Am Nat 109:161-176

Stouffer PC, Kennedy ED, Power HW (1987) Recognition and removal of intraspecific parasite eggs by starlings. Anim Behav 35:1583-1584

Stouffer PC, Romagnano LC, Lombardo MP, Hoffenberg AS, Power HW (1988) A case of communal nesting in the European starling. Condor 90:241-245

Summers-Smith D (1963) The house sparrow. Collins, London

Vehrencamp SL (1977) Relative fecundity and parental effort in communally nesting anis, Crotophaga sulcirostris. Science 197:403-405

Vehrencamp SL (1978) The adaptive significance of communal nesting in groove-billed anis (Crotophaga sulcirostris). Behav Ecol Sociobiol 4:1-33

Verbeyen RF (1980) Breeding strategies of the starlings. In: Wright EN, Iglis IR, Feare CJ (eds) Bird problems in agriculture. British Crop Protection Council Publications, Croydon, UK, pp 69-82

Victoria JK (1972) Clutch characteristics and egg discriminative ability of the African village weaverbird, Ploceus cucullatus. Ibis 114:367-376

Weller MW (1959) Parasitic egg laying in the redhead Aythya americana and other North American Anatidae. Ecol Monogr 29:333-365

Woodward AE, Mather FB (1964) The timing of ovulation, movement of the ovum through the oviduct, pigmentation, and shell deposition in Japanese quail (Coturnix coturnix japonica). Poultry Sci 43:1427-1432

Yom-Tov Y (1980) Intraspecific parasitism in birds. Biol Rev 55:93-108

Yom-Tov Y, Dunnet GM, Anderson A (1974) Intraspecific nest parasitism in the starling, Sturnus vulgaris. Ibis $116: 87-90$

Zar JH (1974) Biostatistical analysis. Prentice Hall, Englewood Cliffs 